\title{
Interactive comment on "Modeling the biogeochemical effects of rotation pattern and field management practices in a multi-crop (cotton, wheat, maize) rotation system: a case study in northern China" by Wei Zhang et al.
}

Wei Zhang et al.

xunhua.zheng@post.iap.ac.cn

Received and published: 16 January 2019

RC2 This manuscript describes a modeling effort to evaluate the biogeochemical effects of optimizing a cotton, wheat, maize rotation and field management practices. This work is within the scope of Biogeosciences. Modeling efforts such as this are important given the difficulty in designing field experiments that adequately capture the appropriate biogeochemical parameters for each treatment. Overall this work is important in increasing our understanding the environmental impacts of management practices. However, one major weakness is a lack of sufficient experimental validation.

Printer-friendly version

Discussion paper 
Specific comments Page 3, lines 67-68 describe the "release potentials of nitrogenous pollutants". It would be helpful to describe these pollutants in the context of agricultural practices (e.g. nitrate leaching, etc.).

Revised. The sentence has been revised as the reviewer suggested. "High nitrogen and water inputs can result in high release potentials of nitrogenous pollutants and induce a series of environmental problems, such as increased nitrate leaching for water pollution" (Please see lines 55-56)

Page 6, Section 2.2: This description of the DNDC95 model is somewhat redundant with the introduction and could either be shortened here or removed from the introduction.

Revised. The description of DNDC in the Introduction has been deleted and the content in Materials and methods has been shortened. "The DNDC95 model used in this study is one of the latest DNDC versions (www.dndc.sr.unh.edu/model/GuideDNDC95.pdf). The model consists of two components with six modules in total. Driven by given primary ecological factors, the former component simulates the field states of a soil-plant system, such as soil chemical and physical status, vegetation growth and organic matter decomposition. Driven by the soil regulating variables yielded by the former component, the latter component simulates the core biogeochemical processes of carbon and nitrogen transformations and physical processes of liquid and gas transportations and thus the annual dynamics of net ecosystem exchanges of carbon dioxide (CO2) (NEE); emissions of methane ( $\mathrm{CH} 4)$, nitrous oxide (N2O), NH3 and NO; and NO3leaching and the inter-annual dynamics of SOC and NEGE. These features enable the model to investigate the integrative biogeochemical effects of the changes in rotation patterns of multiple crops and/or other management practices based on comprehensive validation." (Please see lines 89-99)

Page 8, Section 2.4: Six level-I scenarios are described with increasing the number of cotton rotations with W-M. How were multiple cotton crops incorporated? For R2 was it

Printer-friendly version

Discussion paper
Interactive comment

\section{(}


2 years of cotton followed by 4 years $\mathrm{W}-\mathrm{M}$ or 1 or 2 years of $\mathrm{W}-\mathrm{M}$ between each cotton crop?

Revised. The description of level-I scenarios has been improved to avoid ambiguity as the reviewer suggested. "There were six level-I scenarios in total, hereinafter referred to as $R 0, R 1, \ldots, R 5$, and the number of rotation indicated the years of consecutive cotton planting. For instance, R0 denotes the 6-year monoculture of the W-M; R2 represents the 2-year continuous cotton rotated with the 4-year consecutive W-M; and so on." (Please see lines 142-145)

Page 23, Section 4.3: This discussion of the BMP should also include a discussion of the potential impacts of weed or disease pressure. Continuous cultivation of these crops could lead to challenges for weed or disease management which does not appear to be adequately addressed by the model. This section should include a discussion of these limitations.

Revised. The discussion on weed or disease pressure due to the model limitation has been included as the reviewer suggested. "The first is the possible limitation of the applied model, which cannot simulate the potential effects of monoculture on weed and disease, as well as yields. Continuous cultivation of these crops, especially for cotton, could lead to challenges for weed and disease managements. On the other hand, the scenarios of continuous no-tillage for the W-M could also increase the pressure of weed and disease. But these effects could not be adequately addressed by the current model. Thus, proper parameterization of the effects of monoculture and no-tillage on weed and disease, as well as yields, would be beneficial for screening the more realistic and effective BMP." (Please see lines 430-437)

Page 25: The number of scenarios mentioned in the discussion is different from page 9 and 18.

Revised. Monte Carlo simulation has been applied for BMP selection, thus there are 1000 scenarios for each rotation pattern and 6000 scenarios for BMP selection. "To

Printer-friendly version

Discussion paper
Interactive comment 
screen the BMP of six rotation patterns in interaction with all considered management practices, the variation of fertilizer amount, irrigation amount and residue incorporation rate was set as $40 \%$ of baseline to baseline (N44/172 to N110/430), $40 \%$ of baseline to baseline (I40 to I100) and 0 to $100 \%$ (RI0 to RI100). The factors of irrigated method and tillage consisted of flood (IF) and sprinkle (IS) irrigation, and no-tillage (T0), reduced tillage $(5 \mathrm{~cm}$ and $10 \mathrm{~cm}, \mathrm{~T} 5$ and T10) and conventional tillage $(20 \mathrm{~cm}, \mathrm{~T} 20)$, respectively. We assumed the frequency distribution of all the factors were uniform. Monte Carlo simulations, 1000 combination scenarios of field managements, were used to screen the BMP for each rotation pattern, and the final BMP for the system were selected from the BMPs of six rotation patterns in light of 6000 combination scenarios." (Please see lines 161-170)

Figure 2: It is difficult to differentiate between the different rotation patterns since most of the symbols are stacked. An additional figure or table showing the order of rotations for R0-R5 would be helpful.

Revised. An additional table (Table S4) in supplementary material has been provided to differentiate the different rotation patterns as the reviewer suggested. (Please see the Table S4 in supplementary material)

Technical corrections Line 79: Remove "s" from "contents" Line 132: Replace "its" with "associated" Line 619 should not be indented.

Revised. The sentences have been corrected as the reviewer suggested. (Please see line 63, line 86 and line 430)

Please also note the supplement to this comment: https://www.biogeosciences-discuss.net/bg-2018-401/bg-2018-401-AC2supplement.zip

Interactive comment on Biogeosciences Discuss., https://doi.org/10.5194/bg-2018-401, 2018. 